CONTROL ENGINEERING PRACTICE Vol. 58 p. 343-353, 2017

DOI: 10.1016/j.conengprac.2016.03.021

\title{
Nonlinear controller for bispectral index tracking: Robustness and on-line retuning
}

\author{
Filipa N. Nogueira ${ }^{\mathrm{a}, \mathrm{b}}$, Teresa Mendonça ${ }^{\mathrm{a}, \mathrm{c}}$, Paula Rocha ${ }^{\mathrm{a}, \mathrm{b}}$ \\ ${ }^{a}$ SYSTEC - Research Center for Systems 86 Technologies \\ ${ }^{b}$ Faculdade de Engenharia da Universidade do Porto, Porto, Portugal. \\ ${ }^{c}$ Faculdade de Ciências da Universidade do Porto, Porto, Portugal.
}

\begin{abstract}
In this paper, the performance of a control law designed for the automatic administration of propofol and of remifentanil in order to track a desired level for the bispectral index (BIS), used as a measure of the depth of anesthesia, is analyzed under the presence of model parameters uncertainties. It is theoretically proved and illustrated by simulations that under these circumstances the controller has a very good performance as the BIS converges to a value contained in a neighborhood of the desired BIS level. a retuning strategy in order to improve the BIS tracking under the presence of uncertainties was also theoretically deduced. Simulations show that this strategy leads to BIS tracking improvement. The performance of the controller in clinical environment is illustrated by a clinical case.
\end{abstract}

Keywords:

\footnotetext{
This work was supported by GALENO - Modeling and Control for personalized drug administration, Funding Agency: FCT, Programme: FCT PTDC/SAUBEB/103667/2008. The authors acknowledge Dr. Rui Rabiço (anesthesiologist at Unidade Local de Saúde de Matosinhos - Hospital Pedro Hispano), that assessed the clinical case, and they also acknowledge Dr Manuel Seabra, director of the anesthesia department. Additionally, Filipa Nogueira acknowledges the support of FCT - Portugal through the grant SFRH/BD/48314/2008.
} 
Positive systems, automatic control, robustness, DoA, BIS, anesthesia.

\section{Introduction}

Correct administration of anesthesia drug is crucial for the success of surgery. In this regard, the controllers developed for automatic administration of drugs have to be extensively studied. It is important to study the performance of the controller under the presence of modeling errors as the complex nature of the human body and the inter- and intra-individual variability do not allow obtaining an exact description of the drug effect in each patient. In this sense, it is also crucial to develop retuning techniques to calibrate the controller in order to address possible misfits between the obtained results and the desired values, arising not only from modeling errors, but also from noisy measurements.

Here, the focus is the administration of the hypnotic propofol and of the analgesic remifentanil. These drugs are used for the control of the depth of anesthesia (DoA). The DoA is related to the intensity of two components of general anesthesia: analgesia and hypnosis. According to several studies (Tirén et al. [1], Grindstaff and Tobias [2], Ekman et al. [3], Wodey et al. [4], Whyte and Booker [5]) the DoA may be measured by means of the bispectral index (BIS). This index is a single dimensionless number, which is computed from the electroencephalogram (EEG) and ranges from 0 (equivalent to EEG silence) to 100 (equivalent to fully awake and alert). According to clinical experience, a BIS value between 40 and 60 is desirable for general anesthesia purposes, as it usually corresponds to an adequate state of uncon- 
sciousness during surgical procedures. This is usually achieved manually by the anesthesiologists. However, due to the high complexity of this procedure, an automated system for drug administration would be a good support for the clinicians. This question has deserved the attention of several researchers and led to a number of contributions and controlleres namely a predictive control in Ionescu et al. [6], an adaptive model-based controller in Mortier et al. [7] and Simanski et al. [8], a PID in Padula et al. [9], a neural in Ortolani et al. [10], a fuzzy logic in Shieh et al. [11], a model predictive control in Sawaguchi et al. [12] and Chang et al. [13]. but in these contributions the control of the DoA is not fully automatic. More concretely, the administration of the hypnotic is made automatically, however the administration of the analgesic is made manually by the anesthetic. A detailed introduction to anesthesia as a control problem together with a good overview of the state of the art can be found in Lemos et al. [14] and Dumont [15].

In Nogueira et al. [16] a control law was proposed for the BIS tracking of patients, during general anesthesia, by means of the automatic administration of propofol and of refimentanil. This controller has the advantage of allowing different combinations of drugs in order to obtain the same value for the BIS level, it allows the changing of the desired reference value for the BIS during the surgical procedure, and it is already used in clinical practices, with good performance according to the anesthesiologists. The big difference between this controller and the aforementioned controllers cited above is that: in the referred works the BIS is controlled by the automatic administration of one single drug (the hypnotic) together with the manual administration of the 
other drug (the analgesic), by the anesthesiologist, whereas in Nogueira et al. [16] these two drugs are automatically administered, without any intervention of the anesthesiologist.

The aim of this work is to study the performance of the controller proposed in Nogueira et al. [16] under the presence of model parameters uncertainties. It is theoretically proved that under these circumstances, the BIS level converges to a value contained in a neighborhood of the desired BIS level. This fact allows a retuning strategy in order to recalibrate the controller for BIS tracking improvement. The theoretical deduction of this strategy is here presented.

The structure of this paper is as follows. Section 2 is devoted to the explanation of the BIS model, while the control law is presented in Section 3. In Section 4 the robustness of the controller is analyzed and in Section 5 an on-line retuning strategy is theoretically deduced. Simulations are illustrated in Section 6 while a clinical case is presented in Section 7. Conclusions are drawn in Section 8.

\section{Model description}

The patient BIS level obtained by means of the administration of the hypnotic propofol and of the analgesic remifentanil may be modeled by a new Wiener model recently introduced in the literature Silva et al. [17] and known as the parameter parsimonious model (PPM). According to this model, the linear relations between the propofol and remifentanil dosages and the correspond- 
ing effect concentrations $\left(c_{e}^{p}\right.$ and $\left.c_{e}^{r}\right)$ are modeled by the transfer functions:

$$
\begin{aligned}
& H^{p}(s)=\frac{k_{1} k_{2} k_{3} \alpha^{3}}{\left(k_{1} \alpha+s\right)\left(k_{2} \alpha+s\right)\left(k_{3} \alpha+s\right)} u^{p}(s), \\
& H^{r}(s)=\frac{l_{1} l_{2} l_{3} \eta^{3}}{\left(l_{1} \eta+s\right)\left(l_{2} \eta+s\right)\left(l_{3} \eta+s\right)} u^{r}(s),
\end{aligned}
$$

respectively, where $\alpha$ and $\eta$ are patient dependent parameters, without any explicit physiological meaning, $k_{1}, k_{2}, k_{3}$ and $l_{1}, l_{2}, l_{3}$ are dimensionless constants whose values were identified in Silva et al. [17] from a real patient database, as: $k_{1}=10, k_{2}=9, k_{3}=1, l_{1}=3, l_{2}=2, l_{3}=1$. The complex functions $u^{p}(s)$ and $u^{r}(s)$ are the Laplace transforms of the administered doses of propofol, $u^{p}(t)$, and of remifentanil, $u^{r}(t)$, in $m g \mathrm{~min}^{-1}$. The corresponding BIS level, $z(t)$, usually given by the generalized Hill equation Minto et al. [18], is approximated in Silva et al. [17] by the nonlinear equation:

$$
z(t)=\frac{97.7}{1+U^{\gamma}}
$$

where $U=\mu \frac{c_{e}^{p}}{E C_{50}^{p}}+\frac{c_{e}^{r}}{E C_{50}^{r}}$, and $\mu$ and $\gamma$ are patient dependent parameters, without any physiological meaning, 97.7 is the BIS level at zero concentration, and $E C_{50}^{p}$ and $E C_{50}^{r}$ respectively denote the propofol and remifentanil concentrations that produce half the maximal effect when the drug acts in isolation. The parameters $E C_{50}^{p}$ and $E C_{50}^{r}$ are taken to be fixed, namely $E C_{50}^{p}=10 \mathrm{mg} / \mathrm{ml}$ and $E C_{50}^{r}=0.01 \mathrm{mg} / \mathrm{ml}$. These values were obtained in 
the work developed in Mendonça et al. [19].

The PPM may be also represented by the following state space representation:

$$
\left\{\begin{aligned}
\dot{x}(t) & =A x(t)+B u(t) \\
{\left[\begin{array}{c}
c_{e}^{p}(t) \\
c_{e}^{r}(t)
\end{array}\right] } & =\left[\begin{array}{llllll}
0 & 0 & 1 & 0 & 0 & 0 \\
0 & 0 & 0 & 0 & 0 & 1
\end{array}\right] x(t) \\
U(t) & =C x(t) \\
z(t) & =\frac{97.7}{1+U^{\gamma}},
\end{aligned}\right.
$$

where 


$$
\begin{aligned}
& C=\left[\begin{array}{llllll}
0 & 0 & 0.1 \mu & 0 & 0 & 100
\end{array}\right] \\
& A=\left[\begin{array}{cc}
A^{p} & 0 \\
0 & A^{r}
\end{array}\right], \quad B=\left[\begin{array}{cc}
B^{p} & 0 \\
0 & B^{r}
\end{array}\right], \\
& A^{p}=\left[\begin{array}{ccc}
-10 \alpha & 0 & 0 \\
9 \alpha & -9 \alpha & 0 \\
0 & \alpha & -\alpha
\end{array}\right], \quad A^{r}=\left[\begin{array}{ccc}
-3 \eta & 0 & 0 \\
2 \eta & -2 \eta & 0 \\
0 & \eta & -\eta
\end{array}\right] \text {, } \\
& B^{p}=\left[\begin{array}{c}
10 \alpha \\
0 \\
0
\end{array}\right], \quad B^{r}=\left[\begin{array}{c}
3 \eta \\
0 \\
0
\end{array}\right]
\end{aligned}
$$

This specific form of the state space realization has compartmental structure. This has the advantage of allowing the use of the positive control law defined in the next section.

\section{Controller description}

The nonlinear controller presented in Nogueira et al. [16] was designed for the automatic administration of propofol and of remifentanil in order to control the BIS level of a patient. This control law, which results from a combination of a linear controller with a positivity constraint for the drug doses, is defined by: 


$$
u(t)=\left[\begin{array}{l}
u^{p}(t) \\
u^{r}(t)
\end{array}\right]=\left[\begin{array}{c}
\max \left(0, \tilde{u}^{p}(t)\right) \\
\max \left(0, \tilde{u}^{r}(t)\right)
\end{array}\right]
$$

where $u^{p}$ is the input of propofol and $u^{r}$ is the input of remifentanil, with:

$$
\tilde{u}(t)=\left[\begin{array}{c}
\tilde{u}^{p}(t) \\
\tilde{u}^{r}(t)
\end{array}\right]=E\left(-K A x(t)+\lambda\left(M^{*}-K x(t)\right)\right),
$$

and

$$
\begin{aligned}
& E=\left[\begin{array}{l}
\rho \\
1
\end{array}\right] \frac{1}{\alpha \rho+300 \eta} \\
& M^{*}=\frac{3(0.1 \rho+100)}{0.1 \mu \rho+100}\left(\frac{97.7}{z^{*}}-1\right)^{\frac{1}{\gamma}}, \\
& K=\left[\begin{array}{llllll}
0.1 & 0.1 & 0.1 & 100 & 100 & 100
\end{array}\right]
\end{aligned}
$$

$z^{*}$ is the desired BIS level, and $\lambda$ and $\rho$ are positive design parameters that do not affect the tracked reference value and can be chosen according to clinical criteria. The parameter $\lambda$ influences the convergence speed to the desired reference value and the parameter $\rho$ can be interpreted as the proportion between the doses of propofol and remifentanil.

For more details about this controller and its tracking properties, the reader 
is referred to Nogueira et al. [16].

The robustness of the controller (6) is analyze in the next section.

\section{Controller robustnes in the presence of uncertainties in the lin- ear part of the PPM}

Here it will be proven that the controller (6) is robust under the presence of uncertainties in the linear part of the PPM. More concretely, in this case, the BIS of the patient will converge to a value contained in a neighborhood of the desired reference for the BIS level. This is acceptable from the clinical point of view, as, in clinical practice, the BIS level should be contained in an interval, usually between 40 and 60 .

Instead of the real values for the parameters $\alpha$ and $\eta$, estimates for those values, $\hat{\alpha}$ and $\hat{\eta}$, are respectively used. This implies that instead of using $u$ as described in (11), for the automatic administration of propofol and of remifentanil, the controller $\hat{u}$ as described below is used.

$$
\hat{u}(t)=\left[\begin{array}{c}
\hat{u}^{p}(t) \\
\hat{u}^{r}(t)
\end{array}\right]=\left[\begin{array}{c}
\max \left(0, \hat{\tilde{u}}^{p}(t)\right) \\
\max \left(0, \hat{\tilde{u}}^{r}(t)\right)
\end{array}\right]
$$

with:

$$
\hat{\tilde{u}}=\left[\begin{array}{c}
\hat{\tilde{u}}^{p}(t) \\
\hat{\tilde{u}}^{r}(t)
\end{array}\right]=\hat{E}\left(-K \hat{A} x(t)+\lambda\left(M^{*}-K x(t)\right)\right),
$$

where 


$$
\hat{A}=\left[\begin{array}{cccccc}
-10 \hat{\alpha} & 0 & 0 & 0 & 0 & 0 \\
9 \hat{\alpha} & -9 \hat{\alpha} & 0 & 0 & 0 & 0 \\
0 & \hat{\alpha} & -\hat{\alpha} & 0 & 0 & 0 \\
0 & 0 & 0 & -3 \hat{\eta} & 0 & 0 \\
0 & 0 & 0 & 2 \hat{\eta} & -2 \hat{\eta} & 0 \\
0 & 0 & 0 & 0 & \hat{\eta} & -\hat{\eta}
\end{array}\right],
$$

and

$$
\hat{E}=\left[\begin{array}{l}
\rho \\
1
\end{array}\right] \frac{1}{\hat{\alpha} \rho+300 \hat{\eta}} .
$$

Defining

$$
k=\frac{\alpha \rho+300 \eta}{\hat{\alpha} \rho+300 \hat{\eta}},
$$

the matrix $\hat{E}$ may be written as:

$$
\hat{E}=E k,
$$

and then $\hat{\tilde{u}}$ may be written as

$$
\hat{\tilde{u}}(t)=E k\left(-K \hat{A} x(t)+\lambda\left(M^{*}-K x(t)\right)\right) .
$$

In the sequel, it is proven that when $\hat{u}$ is applied to the PPM, as described 
in (4), the obtained BIS value converges to a steady state value in a neighborhood of the desired BIS level. For this purpose, the auxiliary output

$$
M(x)=K x .
$$

is considered. ${ }^{1}$

In particular, when $M(x)$ converges to $M^{*}$ (as in equation (9)), the BIS level of the patient converges to the reference value $z^{*}$.

Now, let:

$$
\begin{array}{ll}
\Delta_{\alpha}=\alpha-\hat{\alpha}, & r=\max \{8 \hat{\alpha}, \hat{\eta}\}, \\
\Delta_{\eta}=\eta-\hat{\eta}, & s=\max \left\{\left|8 \Delta_{\alpha}\right|,\left|\Delta_{\eta}\right|\right\}
\end{array}
$$

and $\Delta_{K A}=K A-K \hat{A}$,

and let:

$$
I_{M}=\left[\bar{M}_{\min }, \bar{M}_{\max }\right]
$$

with

\footnotetext{
${ }^{1}$ This auxiliary output was used in Nogueira et al. [16], in the context of control without model uncertainties, where it was proven that when $M(x)$ converges to $\bar{M}$, the BIS level converges to $\bar{z}$, with

$$
\bar{z}=\left(\frac{97.7}{1+\left(\frac{0.1 \mu \rho+100}{3(0.1 \rho+100)} \bar{M}\right)^{\gamma}}\right) .
$$




$$
\begin{aligned}
\bar{M}_{\min } & =\frac{k \lambda}{k \lambda+\Delta_{k s}} M^{*}, \\
\bar{M}_{\max } & =\frac{k \lambda}{k \lambda-\Delta_{k s}} M^{*}, \\
\Delta_{k s} & =|1-k| r+s .
\end{aligned}
$$

Applying the LaSalle's invariance principle (see LaSalle [20]) to the Lyapunov function $V: \mathbb{R}^{n} \longrightarrow \mathbb{R}$, described below, it is proven that $M(x)$ converges to $I_{M}$.

$$
V(x)= \begin{cases}\frac{1}{2}\left(M(x)-\bar{M}_{\min }\right)^{2} & \text { if } M(x)<\bar{M}_{\min } \\ \frac{1}{2}\left(M(x)-\bar{M}_{\max }\right)^{2} & \text { if } M(x)>\bar{M}_{\max } \\ 0 & \text { otherwise. }\end{cases}
$$

It will be shown next, by proving that $\dot{V}(x) \leq 0, \forall x \in \mathbb{R}_{+}^{n}$, that the continuous function $V(x)$ is indeed a LaSalle-Lyapunov function of the system on $\mathbb{R}_{+}^{n}$. For this, it has be taken into account that:

$$
\begin{aligned}
& K \hat{A}=-\left[\begin{array}{llllll}
0.1 \hat{\alpha} & 0.1(8 \hat{\alpha}) & 0.1 \hat{\alpha} & 100 \hat{\eta} & 100 \hat{\eta} & 100 \hat{\eta}
\end{array}\right] \\
& \Delta_{K A}= \\
& -\left[\begin{array}{llllll}
0.1 \Delta_{\alpha} & 0.1\left(8 \Delta_{\alpha}\right) & 0.1 \Delta_{\alpha} & 100 \Delta_{\eta} & 100 \Delta_{\eta} & 100 \Delta_{\eta}
\end{array}\right]
\end{aligned}
$$




$$
\begin{gathered}
|K \hat{A}| x \leq r K x=r M(x), \\
\left|\Delta_{K A}\right| x \leq s K x=r M(x) .
\end{gathered}
$$

Since

$$
\dot{V}(x)= \begin{cases}\left(M(x)-\bar{M}_{\min }\right) \dot{M} & \text { if } M(x)<\bar{M}_{\min } \\ \left(M(x)-\bar{M}_{\max }\right) \dot{M} & \text { if } M(x)>\bar{M}_{\max } \\ 0 & \text { otherwise }\end{cases}
$$

with $\dot{M}(x)=K \dot{x}=K A x+K B \hat{u}$, is defined piecewise, the analysis of the non positivity of $\dot{V}(x)$ into two cases has to be made. First it is considered that $M(x)<\bar{M}_{\min }$ and it is proved that in this case $M(x)>0$, implying that $\dot{V}<0$. Secondly, the case when $M(x)>\bar{M}_{\max }$ is studied. In particular, $M(x)<0$ and $\dot{V}<0$ is verified.

Case one $-M(x)<\bar{M}_{\text {min }}$

When $M(x)<\bar{M}_{\text {min }}, M(x)<M^{*}$, and consequently $\hat{\tilde{u}}>0$. Then $\hat{u}=\hat{\tilde{u}}$ and $\dot{M}(x)$ becomes: 


$$
\begin{aligned}
\dot{M}(x) & =K A x+K B \hat{\tilde{u}} \\
& =K A x+\underbrace{K B E}_{1} k\left(-K \hat{A} x+\lambda\left(M^{*}-M(x)\right)\right. \\
& \left.=K A x-k K \hat{A} x+k \lambda\left(M^{*}-M(x)\right)\right) \\
& =K \hat{A} x+\Delta_{K A} x-k K \hat{A} x+k \lambda\left(M^{*}-M(x)\right) \\
& =(1-k) K \hat{A} x+\Delta_{K A} x+k \lambda\left(M^{*}-M(x)\right) \\
& \geq-|1-k||K \hat{A}| x-\left|\Delta_{K A}\right| x+k \lambda\left(M^{*}-M(x)\right) \\
& \geq-|1-k| r M(x)-s M(x)+k \lambda\left(M^{*}-M(x)\right) \\
& =-|1-k| r M(x)-s M(x)+k \lambda M^{*}-k \lambda M(x) \\
& =k \lambda M^{*}-(k \lambda+|1-k| r+s) M(x) \\
& =k \lambda M^{*}-\left(k \lambda+\Delta_{k s}\right) M(x) \\
& =\left(k \lambda+\Delta_{k s}\right)\left(\frac{k \lambda}{k \lambda+\Delta_{k s}} M^{*}-M(x)\right) \\
& =\left(k \lambda+\Delta_{k s}\right)\left(\bar{M}_{m i n}-M(x)\right)>0 .
\end{aligned}
$$

The relation between expressions (36) and (37) is due to the fact that every $a \in \mathbb{R}$ satisfies $a \geq-|a|$, whereas the relation between expressions (37) and (38) results from (28) and (30).

Case two $-M(x)>\bar{M}_{\max }$

Let $M(x)>\bar{M}_{\max }$. If $\hat{\tilde{u}}<0$, then $\hat{u}=0$ and 


$$
\begin{aligned}
\dot{M}(x) & =K A x \\
& =-\left[\begin{array}{llllll}
0.1 \alpha & 0.8 \alpha & 0.1 \alpha & 100 \eta & 100 \eta & 100 \eta
\end{array}\right] x \\
& \leq 0
\end{aligned}
$$

because all the components of $x$ are nonnegative. If $\hat{\tilde{u}}>0$, then $\hat{u}=\hat{\tilde{u}}$ and $\dot{M}(x)$ becomes (see equation (36)):

$$
\begin{aligned}
\dot{M}(x) & =(1-k) K \hat{A} x+\Delta_{K A} x+k \lambda\left(M^{*}-M(x)\right) \\
& \leq|1-k||K \hat{A}| x+\left|\Delta_{K A}\right| x+k \lambda\left(M^{*}-M(x)\right) \\
& \leq|1-k| r M(x)+s M(x)+k \lambda\left(M^{*}-M(x)\right) \\
& =|1-k| r M(x)+s M(x)+k \lambda M^{*}-k \lambda M(x) \\
& =k \lambda M^{*}-(k \lambda-|1-k| r-s) M(x) \\
& =k \lambda M^{*}-\left(k \lambda-\Delta_{k s}\right) M(x) \\
& =\left(k \lambda-\Delta_{k s}\right)\left(\frac{k \lambda}{k \lambda-\Delta_{k s}} M^{*}-M(x)\right) \\
& =\left(k \lambda-\Delta_{k s}\right)\left(\bar{M}_{\max }-M(x)\right)<0,
\end{aligned}
$$

for $k \lambda>\left|\Delta_{k s}\right|$.

Hence, it may been concluded that choosing the parameter $\lambda$ large enough, such that $\lambda>\frac{\left|\Delta_{k s}\right|}{k}, \dot{V}(x)$ is non positive during all time. Then, by the LaSalle's invariance principle, all system trajectories, $x(t)$, converge to the largest set contained in 


$$
W=\left\{x \in \mathbb{R}_{+}^{n}: \dot{V}(x)=0\right\},
$$

which is forward-invariant under the closed-loop dynamics. It follows from (31) that $\dot{V}(x)=0$ either when $u=\tilde{u}$ and $\left(M(x)=\bar{M}_{\min }\right.$ or $M(x)=\bar{M}_{\max }$ or $\left.M(x)=\frac{(1-k) K \hat{A} x+\Delta_{K A} x+k \lambda M^{*}}{k \lambda}\right)$, or when $u=0$, which implies that $M(x)>$ $M^{*}$, and $K A x=0$. Then

$$
\begin{aligned}
W= & I_{1} \cup I_{2} \cup I_{3} \cup I_{4} \text { with } \\
I_{1}=\left\{x \in \mathbb{R}_{+}^{n}: M(x)=\bar{M}_{\text {min }} \text { and } \hat{\tilde{u}}(x)>0\right\} & \\
I_{2}=\{x \in & \left.\mathbb{R}_{+}^{n}: M(x)=\bar{M}_{\text {max }} \text { and } \hat{\tilde{u}}(x)>0\right\} \\
I_{3}=\{x \in & \mathbb{R}_{+}^{n}: M(x)=\frac{(1-k) K \hat{A} x+\Delta_{K A} x}{k \lambda} \\
& \left.\quad+M^{*} \text { and } \hat{\tilde{u}}(x)>0\right\} \\
I_{4}=\{x \in & \left.\mathbb{R}_{+}^{n}: K A x=0 \text { and } \hat{u}(x)=0\right\} .
\end{aligned}
$$

The set $I_{4}$ is not invariant. In fact, if $\hat{u}$ would remain equal to zero, at a certain time instant, $M(x)$ would become smaller than $M^{*}, \hat{\tilde{u}}(x)$ would become positive, and $\hat{u}(x)$ would equal $\hat{\tilde{u}}(x)$, so the trajectory $x(t)$ would leave the subset. On the other hand, both $I_{1}, I_{2}$ and $I_{3}$ are subsets of $I_{M}$, which is invariant, indeed if $M(x) \leq \bar{M}_{\min }$, then $\dot{M}(x) \geq 0$ and if $M(x) \geq \bar{M}_{\max }$, then $\dot{M}(x) \leq 0$. Therefore, one may conclude that $M(x)$ converges to (a subset contained in) the interval $I_{M}$ as previously claimed.

Since the patient BIS response to the administered drug doses $\hat{u}$ is a decreasing function of $M(x)$ (cf. equation (18)), the BIS level converges to the interval: 


$$
\left.I_{B I S}=\right] B I S_{\min }, B I S_{\max }[
$$

with

$$
\begin{aligned}
B I S_{\text {min }} & =\frac{97.7}{1+\left(\frac{0.1 \mu \rho+100}{3(0.1 \rho+100)} \bar{M}_{\text {max }}\right)^{\gamma}} \text { and } \\
B I S_{\text {max }} & =\frac{97.7}{1+\left(\frac{0.1 \mu \rho+100}{3(0.1 \rho+100)} \bar{M}_{\text {min }}\right)^{\gamma}} .
\end{aligned}
$$

As expected, the desired steady state value $z^{*}$ is not achieved, but the patient's BIS remains in a neighborhood of this target value. Moreover, when the errors in the parameters go to 0 , and hence $s$ goes to 0 and $k$ goes to 1 (see the remark below), $\bar{M}_{\min }, \bar{M}_{\max }$ and $M(x)$ converge to $M^{*}$. This implies that the patient BIS converges to the desired value $z^{*}$, which means that the control law is robust with respect to parameter uncertainties. Moreover, as can be seen by expressions (22) and (23), increasing the parameter $\lambda$ decreases the width of the interval $I_{M}$ and consequentely the robustness of the controller is also increased.

Note that:

$$
\begin{aligned}
k & =\frac{\alpha \rho+300 \eta}{\hat{\alpha} \rho+300 \hat{\eta}} \\
& =\frac{\hat{\alpha} \rho+\Delta_{\alpha} \rho+300 \hat{\eta}+300 \Delta_{\eta}}{\hat{\alpha} \rho+300 \hat{\eta}} \\
& =1+\frac{\Delta_{\alpha} \rho+300 \Delta_{\eta}}{\hat{\alpha} \rho+300 \hat{\eta}}
\end{aligned}
$$


thus, if $\Delta_{\alpha}$ and $\Delta_{\eta}$ converge to zero $k$ clearly converges to the value 1 .

As it shall be next proven, in the presence of uncertainties in the PPM linear part, the BIS of the patient not only remains in the interval $I_{B I S}$, but also converges to a specific value $\bar{z} \in I_{B I S}$.

\section{Retuning improvement in the presence of uncertainties in the PPM linear part}

In Nogueira et al. [21], a retuning strategy was proposed in order to overcome the discrepancy between the obtained BIS level for the patient and the desired one. As already mentioned this may be the result of modeling errors and measurement noise. This strategy consists of computing another reference value for $M^{*}$ in (7), by assuming that, in spite of the presence of modeling errors, the BIS converges to a determined value and assuming also that there is a proportion between the obtained BIS value and the reference value $M^{*}$.

Here the specific case where the misfit between the desired BIS value and the obtained one is due to the presence of errors in the PPM linear part parameters is analyzed. Moreover, it is theoretically proven that the patient BIS indeed converges to a specific value $\bar{z}$, which allows the computation of a new reference for $M^{*}$ for recalibration of the control law (16). More concretely, it will be proven that the auxiliary output $M(x)$ will converge to a value $\bar{M}$, which is equivalent to have the BIS converging to the value $\bar{z}=\left(\frac{97.7}{1+\left(\frac{0.1 \mu \rho+100}{3(0.1 \rho+100)} \bar{M}\right)^{\gamma}}\right)$, and then the controller is retuning based on the BIS 
obtained.

In order to prove that $M(x)$ converges to a value $\bar{M}$, it will be first proved that after a certain instant $t_{\varepsilon}$, $\hat{\tilde{u}}$ will remain nonnegative. Then, it will be proved that, in this case, there is only one equilibrium point. After finding the equilibrium point the controller may be recalibrated.

Prove that: $\forall t \geq t_{\varepsilon}, \hat{\tilde{u}}(t) \geq 0$.

Let $\varepsilon \geq 0$. It is intended to prove that $\forall \varepsilon \geq 0, \exists t_{\varepsilon} \geq 0: \hat{\tilde{u}}\left(t_{\varepsilon}\right) \geq 0$.

In the previous section, it was proved that under the presence of uncertainties in the linear part of the PPM, the auxiliary output $M(x)$ remains in the interval $I_{M}$, i.e.,

$$
M(x) \in I_{M}=\left[\bar{M}_{\min }, \bar{M}_{\max }\right]
$$

Thus, for every $\varepsilon \geq 0$ :

$$
M(x) \in I_{M}=\left[\bar{M}_{\min }-\varepsilon, \bar{M}_{\max }+\varepsilon\right]
$$

Note that 


$$
\begin{aligned}
\hat{\tilde{u}} & \geq 0 \\
& \Leftrightarrow \underbrace{E k}_{>0}\left(-K \hat{A} x+\lambda\left(M^{*}-M(x)\right) \geq 0\right. \\
& \Leftrightarrow-K \hat{A} x+\lambda M^{*}-\lambda M(x) \geq 0 \\
& \Leftrightarrow K \hat{A} x+\lambda M(x) \leq \lambda M^{*} .
\end{aligned}
$$

By (28), the following inequalities hold

$$
\begin{aligned}
K \hat{A} x+\lambda M(x) & \leq r M(x)+\lambda M(x) \\
& =(r+\lambda) M(x) \\
& <r M(x) \\
& <r\left(\bar{M}_{\max }+\varepsilon\right) .
\end{aligned}
$$

Consider (see (71) and (75)):

$$
r\left(\bar{M}_{\max }+\varepsilon\right) \leq \lambda M^{*},
$$

which is equivalent to

$$
\varepsilon \leq \frac{\lambda M^{*}}{r}-\bar{M}_{\max }
$$

Thus, for $t \geq t_{\varepsilon}, \lambda>0$ and $\varepsilon=\frac{\lambda M^{*}}{r}-\bar{M}_{\max }, \hat{\tilde{u}}(t) \geq 0$. 
Prove that for all $t \geq t_{\varepsilon}$, there is one equilibrium point.

According to the demonstration conducted above, there exists a certain instant $t_{\varepsilon}>0$, such that for $t \geq t_{\varepsilon}, \hat{u}(t)=\hat{\tilde{u}}(t) \geq 0$. Thus, for $t \geq t_{\varepsilon}$,

$$
\begin{aligned}
\dot{M}(x)=0 & \Leftrightarrow \\
K \dot{x}=0 & \Leftrightarrow K A x+\underbrace{K B E}_{=1} k\left(-K \hat{A} x+\lambda\left(M^{*}-M(x)\right)\right)=0 \\
& \Leftrightarrow K A x-k K \hat{A} x+k \lambda M^{*}-k \lambda M(x)=0 \\
& \Leftrightarrow-K A x+k K \hat{A} x+k \lambda M(x)=k \lambda M^{*}
\end{aligned}
$$

and

$$
\begin{aligned}
\hat{\tilde{u}} & =E\left(-k K \hat{A} x+k \lambda M^{*}-k \lambda M(x)\right) \\
& =E(-k K \hat{A} x-K A x+k K \hat{A} x+k \lambda M(x)-k \lambda M(x)) \quad[\text { by (81) ] } \\
& =-E K A x .
\end{aligned}
$$

When $M(x)=M^{*}$, i.e, when $\dot{M}=0$, the control $\hat{u}$ is then given by $\hat{\tilde{u}}=$ $-E K A x$, and the closed-loop system can be described by

$$
\dot{x}=(A-B E K A) x .
$$

In Nogueira et al. [16] it was shown that the system (85) is stable with the equilibrium point, $x^{e q}$, described by: 


$$
x^{e q}=\left[\begin{array}{c}
\rho \\
\rho \\
\rho \\
1 \\
1 \\
1
\end{array}\right] c_{e}^{r}
$$

where $c_{e}^{r}$ is the effect concentration of remifentanil.

Thus, by (81) and (86), the solution of the system

$$
\left\{\begin{array}{l}
A x+B \hat{u}=0 \\
\dot{M}(x)=0
\end{array}\right.
$$

is computed by the following equation:

$$
-K A\left[\begin{array}{c}
\rho \\
\rho \\
\rho \\
1 \\
1 \\
1
\end{array}\right] \bar{c}_{e}^{r}+k K \hat{A}\left[\begin{array}{c}
\rho \\
\rho \\
\rho \\
1 \\
1 \\
1
\end{array}\right] \bar{c}_{e}^{r}+k \lambda K\left[\begin{array}{l}
\rho \\
\rho \\
\rho \\
1 \\
1 \\
1
\end{array}\right] \bar{c}_{e}^{r}=k \lambda M^{*}
$$

which is equivalent to:

$$
\bar{c}_{e}^{r}=S M^{*}
$$


for suitable $S$, that may be determined by observing the BIS value of the patient. More concretely, and noting that the value of $M^{*}$ is known, after the effect concentration of remifentanil, $\bar{c}_{e}^{r}$, be computed by inverting the Hill equation, $S$ may be determine by the following expressions:

$$
\begin{aligned}
S & =\frac{\bar{c}_{e}^{r}}{M^{*}} \\
& =\frac{\bar{U}}{0.1 \mu \rho+100} \frac{1}{M^{*}} \\
& =\frac{\left(\frac{97.7}{\bar{z}}-1\right)^{\frac{1}{\gamma}}}{0.1 \mu \rho+100} \frac{1}{M^{*}},
\end{aligned}
$$

where $\bar{z}$ is the BIS of the patient and $\bar{U}$ is the corresponding potency.

In order to improve the convergence of the BIS, the reference value $M^{*}$ in the controller (16) is replaced for another reference value $M^{* *}$. The reference $M^{* *}$ must be such that the effect concentration of remifentanil, $\overline{\bar{c}}_{e}^{r}$, obtained by using it would be equal to the desired effect concentration of remifentanil, $c_{e}^{r *}$, i.e., the following equality must hold:

$$
\overline{\bar{c}}_{e}^{r}=c_{e}^{r *}=\frac{M^{*}}{3(0.1 \rho+100)}
$$

By (89), this would be equivalent to:

$$
\overline{\bar{c}}_{e}^{r}=S M^{* *}=\frac{M^{*}}{3(0.1 \rho+100)} .
$$


Which means that

$$
\begin{aligned}
M^{* *} & =\frac{1}{S} \frac{M^{*}}{3(0.1 \rho+100)} \\
& =\frac{0.1 \mu \rho+100}{\left(\frac{97.7}{\bar{z}}-1\right)^{\frac{1}{\gamma}}} M^{*} \frac{M^{*}}{3(0.1 \rho+100)} \\
& =\frac{1}{\left(\frac{97.7}{\bar{z}}-1\right)^{\frac{1}{\gamma}}} M^{*} \frac{0.1 \mu \rho+100}{3(0.1 \rho+100)} M^{*} \\
& =\frac{\left(\frac{97.7}{z^{*}}-1\right)^{\frac{1}{\gamma}}}{\left(\frac{97.7}{\bar{z}}-1\right)^{\frac{1}{\gamma}}} M^{*} .
\end{aligned}
$$

Concluding, in the presence of uncertainties in the linear part of the PPM, the BIS of the patient will converge to a certain value $\bar{z}$, instead of converging to the desired one $z^{*}$. This problem may be overcome, by observing the BIS at steady-state, $\bar{z}$, and retuning the control law replacing the reference value $M^{*}$ for the new reference value $M^{* *}$ computed as in (98).

It is noteworthy that the formula (98) here deduced to compute the reference $M^{* *}$ is the same as the one proposed in Nogueira et al. [21]. This is due to the fact that, although it was not proved in the work presented in Nogueira et al. [21], it was assumed that the BIS converges to a single value and that there is a proportion between the obtained BIS value $\bar{z}$ and the reference BIS value $M^{* *}$, which actually happens when there are errors in the identification of the parameters of the linear part of the PPM, as it was proved in this work. 
Remark 5.1. In clinical practice, sometimes the reference value for the BIS must be changed during surgery. The control law (6) allows this changing by computing another value for $M^{*}$ to be used in the controller, i.e., it is computed $M^{*}=M_{\text {mod }}^{\text {ref2 }}=\frac{3(0.1 \rho+100)}{0.1 \mu \rho+100} \sqrt[r]{\frac{z_{0}}{z_{2}^{*}}-1}$, where $z_{2}^{*}$ is the new reference value for the BIS. In case there is a misfit in the BIS response, a new retuning for the $M^{*}$ value is necessary in order to achieve the new desired reference value for the BIS, which may delay reference tracking. An alternative to overcome this drawback is to immediately use a retuned value for $M^{*}$, given by

$$
M^{*}=\frac{\left(\frac{97.7}{z^{*}}-1\right)^{\frac{1}{\gamma}}}{\left(\frac{97.7}{\bar{z}}-1\right)^{\frac{1}{\gamma}}} M_{m o d}^{r e f 2}
$$

This corresponds to using equation (98), with $M^{*}$ replaced by the new reference value $M_{\text {mod }}^{\text {ref } 2}$.

\section{Simulations}

In this section, the performance of the controller (6), proposed in Nogueira et al. [16], under the presence of uncertainties in the parameters of the linear part of the PPM and the performance of the retuning strategy here proposed are illustrated by means of simulations. Note that although the presented models and control law are in continuous time, they are discretized for implementation (by means of a zoh).

The simulated patients were set up based on the data of real patients subjected to general anesthesia under propofol and remifentanil administration. 
The DoA was monitored by the BIS level and was manually controlled around clinically accepted values by the anesthetist. Alaris GH pumps were used for both propofol and remifentanil. Infusion rates, BIS values and other physiological variables were acquired every five seconds (Mendonça et al. [19]).

In Figure 1 the BIS evolution of a patient is illustrated where the test patient was considered to be modeled by a PPM with realistic parameters: $\alpha=0.086, \eta=0.021, \mu=1.42$, and $\gamma=0.98$ (see Mendonça et al. [19]). On the other hand, the control law (11) was tuned with the design parameters $\lambda=100$ and $\rho=900$, and was tuned for the estimated parameters $\hat{\alpha}=50 \alpha$ and $\hat{\eta}=50 \eta$. The desired reference value for the BIS, $z^{*}$, was set to be 50 . As theoretically proved, the BIS converged to a value $\bar{z}$ that belongs to the interval

$$
\left.I_{B I S}=\right] B I S_{\min }, B I S_{\max }[
$$

with $B I S_{\min }=42$ and $B I S_{\max }=56$. As it may observed, in Figure 1, the BIS response of the patient converged to 54 rather than to the desired reference value, $z^{*}=50$, i.e., the BIS was affected by an error of $8 \%$. The new reference $M^{*}$ was computed, as in (98), at time $t_{0}=40 \mathrm{~min}$, improving the BIS response of this patient, which converged to 48.5 representing an error of $3 \%$. 


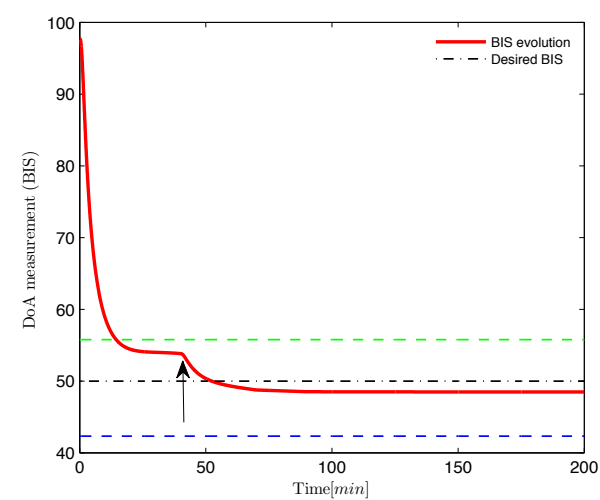

Figure 1: BIS evolution in the presence of uncertainties, $\hat{\alpha}=\mathbf{5 0} \alpha, \hat{\eta}=\mathbf{5 0} \eta$. The reference value for the BIS level was set to be 50 .

In order to test the performance of the retuning strategy under more adverse circumstances, six patients are simulated by six PK/PD Wiener models (other than the PPM) described in Appendix A. The nonlinear part was taken to coincide with the generalized Hill equation (3) and the corresponding parameters $\gamma$ and $\mu$ were identified in Mendonça et al. [19] from the surgery data (see Table B.3). The values of $E C_{50}^{p}$ and $E C_{50}^{r}$ are respectively $10 \mathrm{mg} / \mathrm{ml}$ and $0.01 \mathrm{mg} / \mathrm{ml}$, as previously mentioned. It was also added to the BIS signal Gaussian white noise with zero mean and standard deviation $\sigma_{\text {noise }}=3$. In order to improve the performance of the control procedure in the presence of noise, a filter was applied to the noisy BIS signal. The misfit in the nonlinear part of the patient model was also increased. More concretely, instead of the values $\gamma$ and $\mu$, identified for the six patients (see Table B.3), other values for $\gamma$ and $\mu$ were chosen in the PPM, namely $\gamma=1.88$ and $\mu=1.79$. These values are the average of the values for $\gamma$ and 
$\mu$ taken from a bank of identified values for eighteen real patients obtained in the work developed in Mendonça et al. [19]. As can be seen in Fig. 2 even in this adverse circumstance, the control of the BIS of the patient, with the retuning strategy applied at time instant $t=30 \mathrm{~min}$ also improved the tracking of the desired reference value, which was set to be $50, z^{*}=50$, and was a success from the clinical point of view, more concretely, $100 \%$ of the BIS value obtained were within 40 and 60 , as desired.

The performance of the retuning strategy was also tested under extreme adversities. One hundred patients were simulated with PK/PD Wiener models (other than the PPM) described in Appendix A and were controlled with the control law as described in Nogueira et al. [16], using the PPM. In order to increase the misfit between the patient model and the PPM (used in the controller), the parameters $\alpha, \eta, \mu$ and $\gamma$ were not identified. Instead, we tune the controller with $\alpha=0.0759, \eta=0.5825, \mu=1.79$ and $\gamma=1.88$, for all the one hundred simulations. These values are the average values from a bank of eighteen real patients identified in Mendonça et al. [19]. It was also added to the BIS signal Gaussian white noise with zero mean and standard deviation $\sigma_{\text {noise }}=3$. A filter was then applied to the noisy BIS signal. The desired reference value was set to be $50, z^{*}=50$, and the retuning strategy was applied at time instant $t=30 \mathrm{~min}$. The application of the retuning strategy improved the tracking of the desired reference value in $100 \%$ of the cases, as theoretically expected. Moreover, the BIS obtained after the controller retuning was within 40 and 60 in $100 \%$ of the cases, as clinically desired. The results of these simulations are illustrated in Fig. 3. 

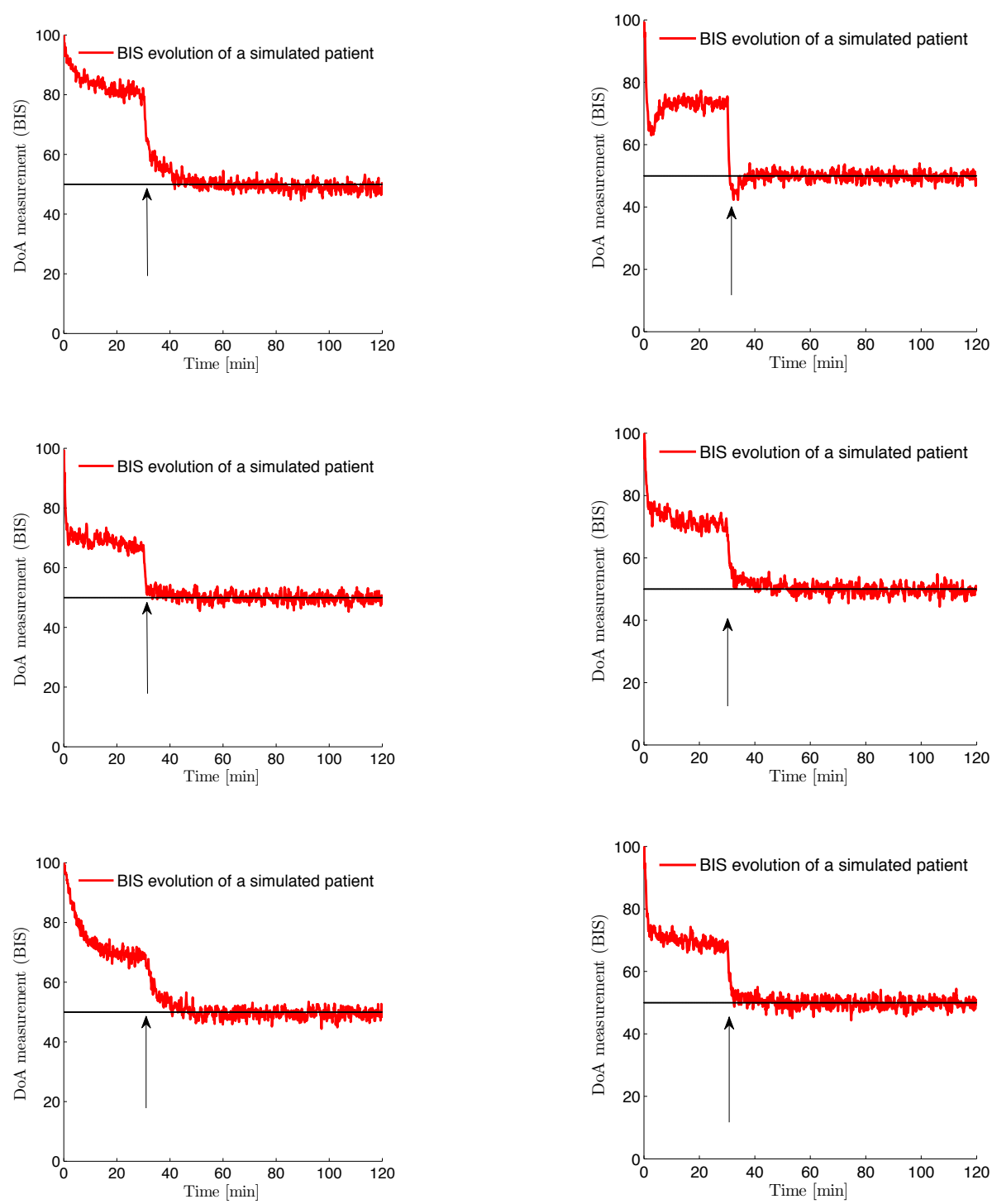

Figure 2: BIS evolution, in the presence of noise, of 6 patients modeled by a $\mathrm{PK} / \mathrm{PD}$ model with a retuning strategy applied at instant $t=30 \mathrm{~min}$. The desired BIS level is set to be 50 . 


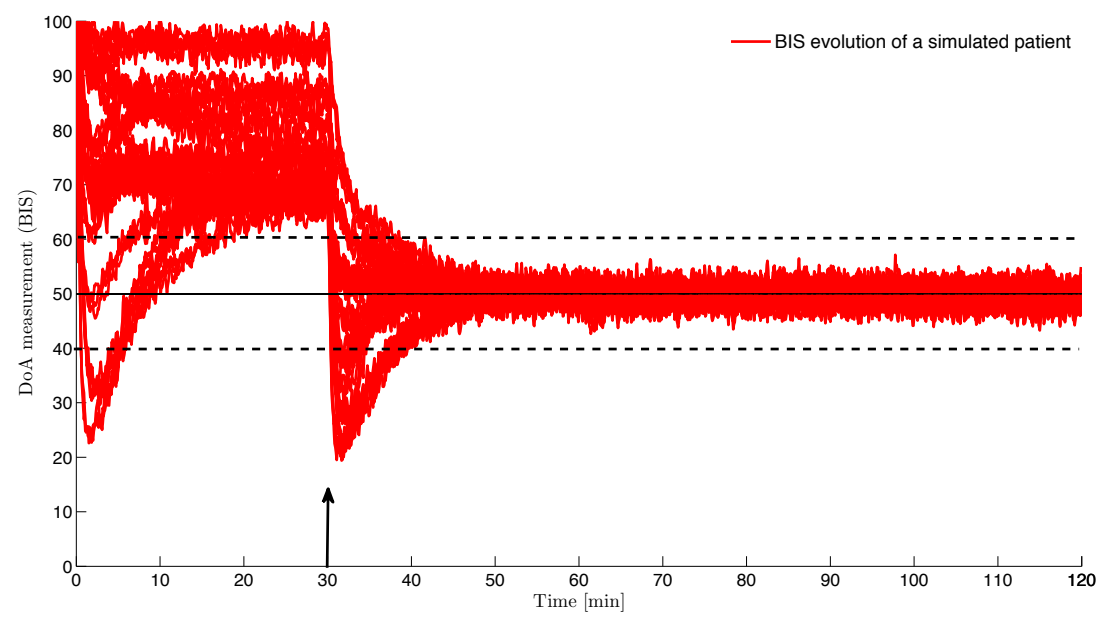

Figure 3: BIS evolution, in the presence of noise, of 100 patients modeled by a $\mathrm{PK} / \mathrm{PD}$ model with a retuning strategy applied at instant $t=30 \mathrm{~min}$. The desired BIS level is set to be 50 .

\section{Clinical case}

In this section, a clinical case is presented where the administration of propofol and remifentanil was automatically made by using the controller proposed in Nogueira et al. [16], integrated in the Galeno platform (Costa et al. [22]). This platform was developed in the framework of the portuguese funding agency (FCT) project Galeno, and incorporates several identification and control procedures for automation in anesthesia. This supervisory automatic drug administration system is currently implemented in a surgery room at the ULSM (Pedro Hispano Hospital, Matosinhos, Portugal), where the data here presented were collected, under medical surveillance. Manual drug administration is ready to be switched on both under clinical decision or in 
case of failure of the automatic controller. The patient, a man of 85 years of age, $80 \mathrm{~kg}$ of weight and $1.72 \mathrm{~m}$ of height was subject to general anesthesia, for a partial gastrectomy. Alaris GH pumps were used for both propofol and remifentanil. Infusion rates, BIS values and other physiological variables were acquired with a sampling time of five seconds. The neuromuscular blockade (NMB) was controlled manually by bolus administration.

Due to clinical constraints associated to the anesthetic procedures adjusted to the patient and also for clinical constraints, the controller was not started at the beginning of the anesthetic procedure.

In Figure 4 a summary of the global situation that highlights the main features of the controller and the relevant anesthetics clinical data during the surgery is presented. The time for initialization of the automatic controller was defined by the anesthetists and is marked with a red arrow.

The desired BIS level was initially set to be 50 and was changed along the surgery (at time instants: $t_{1}=8 \mathrm{~min}, t_{2}=47 \mathrm{~min}, t_{3}=55 \mathrm{~min}$, $t_{4}=95 \mathrm{~min}$ ), due to clinical directives. As it can be observed, the automatic controller presented an adequate clinical behavior leading to the desired reference tracking.

According to the anesthesiologists, the performance of the controller is considered to be satisfactory. 


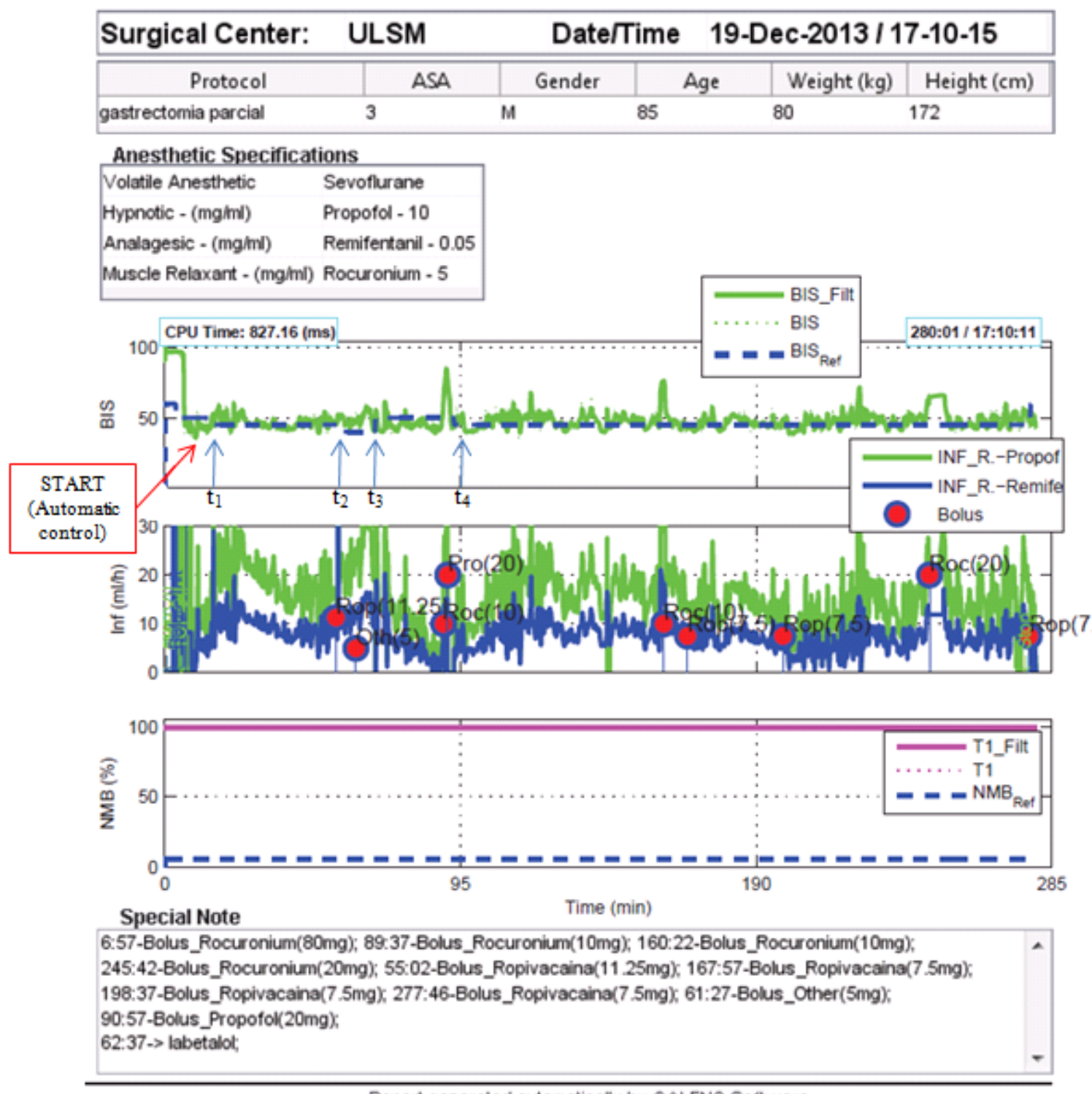

Report generated automatically by GALENO Software

Figure 4: Partial printout of the final report of the clinical case. The dashed blue row on the top graph corresponds to the desired reference value for the BIS. The top red arrow marks the initialization of the automatic control. The NMB plot should be disregarded, as this feature is being manually controlled. 


\section{Conclusion}

In this paper, the performance of a nonlinear controller, proposed recently in the literature, for automatic administration of propofol and of remifentanil in order to control the BIS of a patient, was analyzed. Here, it was theoretically proven that under the presence of uncertainties in the parameters of the linear part of the BIS model, the BIS of the patient converges to a value that is contained in a neighborhood of the desired BIS level. A retuning strategy to recalibrate the controller in order to improve the BIS tracking under the presence of uncertainties was also theoretically deduced. The robustness theoretical analysis and the performance of the retuning strategy here proposed were illustrated by simulations. Moreover, the performance of the controller in clinical practice was also illustrated. Both the simulations and the clinical case have shown that the controller has a good performance under the presence of parameter uncertainties and that the retuning strategy here proposed improves the tracking for the desired reference. These results, together with the positive global assessment of the anesthesiologists concerning the controller features, constitute a strong encouragement to consider the proposed controller as good option for use in clinical environment.

\section{Appendix A. PK/PD Model Description}

The effect concentration of propofol $\left(c_{e}^{p}\right)$ and of remifentanil $\left(c_{e}^{r}\right)$ can be modeled by the PK/PD state space models given in Ionescu et al. [23], based on the material presented in Bailey and Haddad [24], Marsh et al. [25], Minto et al. [26], and Schnider et al. [27], which have the following form: 
where

$$
\left\{\begin{array}{l}
\dot{x^{i}}=A^{i} x^{i}+B^{i} u^{i} \\
c_{e}^{i}=\left[\begin{array}{llll}
0 & 0 & 0 & 1
\end{array}\right] x^{i},
\end{array}\right.
$$

$i=p, r ; \quad p$ stands for propofol and $r$ stands for remifentanil,

$$
x^{i}=\left[\begin{array}{r}
x_{1}^{i} \\
x_{2}^{i} \\
x_{3}^{i} \\
x_{4}^{i}
\end{array}\right]
$$

$$
A^{i}=\left[\begin{array}{cccc}
-k_{10}^{i}-k_{12}^{i}-k_{13}^{i} & k_{21}^{i} V_{2}^{i} / V_{1}^{i} & k_{31}^{i} V_{3}^{i} / V_{1}^{i} & 0 \\
k_{12}^{i} V_{1}^{i} / V_{2}^{i} & -k_{21}^{i} & 0 & 0 \\
k_{13}^{i} V_{1}^{i} / V_{3}^{i} & 0 & -k_{31}^{i} & 0 \\
k_{e 0}^{i} & 0 & 0 & -k_{e 0}^{i}
\end{array}\right] \text {, }
$$

$$
B^{i}=\left[\begin{array}{c}
\frac{1}{V_{1}^{i}} \\
0 \\
0 \\
0
\end{array}\right]
$$


with, for the case of propofol $(i=p)$ :

$$
\begin{aligned}
& V_{1}^{i}=4.27[l] \\
& V_{2}^{i}=18.9-0.391(\text { age }-53)[l] \\
& V_{3}^{i}=238[l] \\
& C_{1}^{i}=1.89+0.0456(\text { weight }-77)-0.0681(\text { lbm }-59)+0.0264(\text { height }-177)[l / m] \\
& C_{2}^{i}=1.29-0.024(\text { age }-53)[l / m] \\
& C_{3}^{i}=0.836[l / m] \\
& K_{e 0}^{i}=0.456\left[\mathrm{~min}^{-1}\right] \\
& K_{10}^{i}=\frac{C_{1}^{i}}{V_{1}^{i}}\left[\mathrm{~min}^{-1}\right] \\
& K_{12}^{i}=\frac{C_{2}^{i}}{V_{1}^{i}}\left[\mathrm{~min}^{-1}\right] \\
& K_{13}^{i}=\frac{C_{3}^{i}}{V_{1}^{i}}\left[\mathrm{~min}^{-1}\right] \\
& K_{21}^{i}=\frac{C_{2}^{i}}{V_{2}^{i}}\left[\mathrm{~min}^{-1}\right] \\
& K_{31}^{i}=\frac{C_{3}^{i}}{V_{3}^{i}}\left[\mathrm{~min}^{-1}\right]
\end{aligned}
$$


and, for the case of remifentanil $(i=r)$ :

$$
\begin{aligned}
& V_{1}^{i}=5.1-0.0201(\text { age }-40)+0.072(\mathrm{lbm}-55)[l] \\
& V_{2}^{i}=9.82-0.0811(\text { age }-40)+0.108(\mathrm{lbm}-55)[l] \\
& V_{3}^{i}=5.42[l] \\
& C_{1}^{i}=2.6-0.0162(\text { age }-40)+0.0191(\mathrm{lbm}-55)[\mathrm{l} / \mathrm{m}] \\
& C_{2}^{i}=2.05-0.0301(\text { age }-40)[\mathrm{l} / \mathrm{m}] \\
& C_{3}^{i}=0.076-0.00113(\text { age }-40)[\mathrm{l} / \mathrm{m}] \\
& K_{e 0}^{i}=0.595-0.007(\text { age }-40)\left[\mathrm{min}^{-1}\right] \\
& K_{10}^{i}=\frac{C_{1}^{i}}{V_{1}^{i}}\left[\mathrm{~min}^{-1}\right] \\
& K_{12}^{i}=\frac{C_{2}^{i}}{V_{1}^{i}}\left[\mathrm{~min}^{-1}\right] \\
& K_{13}^{i}=\frac{C_{3}^{i}}{V_{1}^{i}}\left[\mathrm{~min}^{-1}\right] \\
& K_{21}^{i}=\frac{C_{2}^{i}}{V_{2}^{i}}\left[\mathrm{~min}^{-1}\right] \\
& K_{31}^{i}=\frac{C_{3}^{i}}{V_{3}^{i}}\left[\mathrm{~min}^{-1}\right]
\end{aligned}
$$

The lean body mass $(l \mathrm{lbm})$ for women and men are computed, respectively, by the equations

$$
1.07 \text { weight }-148 \frac{\text { weight }^{2}}{\text { height }^{2}} \text { and } 1.1 \text { weight }-128 \frac{\text { weight }^{2}}{\text { height }^{2}} \text {. }
$$

\section{Appendix B. Database}

This database was courteously provided by Galeno project (http://www2.fc.up.pt/galeno/). 
The parameters presented in Table B.2 were identified in Mendonça et al. [19].

Table B.1: Patient features

\begin{tabular}{lcccc}
\hline & Gender & Age & Height $(\mathbf{c m})$ & Weight (kg) \\
\hline Patient 1 & F & 56 & 160 & 88 \\
Patient 2 & F & 64 & 146 & 60 \\
Patient 3 & F & 29 & 163 & 59 \\
Patient 4 & F & 51 & 163 & 55 \\
Patient 5 & F & 68 & 158 & 113 \\
Patient 6 & F & 66 & 155 & 74 \\
\hline
\end{tabular}

Table B.2: PPM parameters

\begin{tabular}{ccc}
\hline & $\alpha$ & $\eta$ \\
\hline Patient 1 & 0.0667 & 0.3989 \\
Patient 2 & 0.0489 & 0.1269 \\
Patient 3 & 0.0737 & 0.2793 \\
Patient 4 & 0.0701 & 0.2837 \\
Patient 5 & 0.0687 & 4.5413 \\
Patient 6 & 0.1336 & 0.2307 \\
\hline
\end{tabular}


Table B.3: PK/PD model parameters - Hill eq.

\begin{tabular}{ccc}
\hline & $\gamma$ & $\mu$ \\
\hline Patient 1 & 2.0321 & 4.3266 \\
Patient 2 & 1.0702 & 3.9505 \\
Patient 3 & 3.7297 & 4.1494 \\
Patient 4 & 1.8645 & 3.8367 \\
Patient 5 & 0.9882 & 3.8094 \\
Patient 6 & 1.5613 & 4.2411 \\
\hline
\end{tabular}

\section{References}

[1] C. Tirén, R. Anderson, G. Barr, A. Owall, J. Jakobsson, Clinical comparison of three different anaesthetic depth monitors during cardiopulmonary bypass, Anaesthesia 60 (2005) 189-193.

[2] R. Grindstaff, J. Tobias, Applications of bispectral index monitoring in the pediatric intensive care unit, J Intensive Care Med 19 (2004) $111-116$.

[3] A. Ekman, M. Lindholm, C. Lennmarken, R. Sandin, Reduction in the incidence of awareness using bis monitoring, Acta Anaesthesiol Scand 48 (2004) 20-26.

[4] E. Wodey, O. Tirel, J. Y. Bansard, A. Terrier, C. Chanavaz, R. Harris, C. Ecoffey, L. Senhadji, Impact of age on both bis values and eeg bis- 
pectrum during anaesthesia with sevoflurane in children, Br J Anaesth 94 (2005) 810-820.

[5] S. D. Whyte, P. D. Booker, Monitoring depth of anaesthesia by eeg, British Journal of Anaesthesia 3 (2003) 106-110.

[6] C. M. Ionescu, R. De Keyser, B. C. Torrico, T. De Smet, M. M. Struys, J. E. Normey-Rico, Robust predictive control strategy applied for propofol dosing using bis as a controlled variable during anesthesia, IEEE Transactions on Bio-medical Engineering 55 (2008) 2161-2170.

[7] E. Mortier, M. Struys, T. De Smet, L. Versichelen, G. Rolly, Closed-loop controlled administration of propofol using bispectral analysis, Anaesthesia 53 (1998) 749-754.

[8] O. Simanski, A. Sievert, M. Janda, J. Bajorat, Current developments in automatic drug delivery in anesthesia, Biomedizinische Technik/Biomedical Engineering (2013).

[9] F. Padula, C. Ionescu, N. Latronico, M. Paltenghi, A. Visioli, G. Vivacqua, A gain-scheduled pid controller for propofol dosing in anesthesiaoriginal research article, Proceedings of the 9th IFAC Symposium on Biological and Medical Systems (2015) 545 - 550 .

[10] O. Ortolani, A. Conti, A. D. Filippo, C. Adembri, E. Moraldi, A. Evangelisti, M. Maggini, S. J. Roberts, Eeg signal processing in anaesthesia. use of a neural network technique for monitoring depth of anaesthesia, British Journal of Anaesthesia 88 (2002) $644-648$. 
[11] J. S. Shieh, D. A. Linkens, A. J. Asbury, A hierarchical system of online advisory for monitoring and controlling the depth of anaesthesia using self-organizing fuzzy logic, Engineering Applications of Artificial Intelligence 18 (2005) $307-316$.

[12] Y. Sawaguchi, E. Furutani, G. Shirakami, M. Araki, K. Fukuda, A model-predictive hypnosis control system under total intravenous anesthesia, IEEE Transactions on Biomedical Engineering 55 (2008) 874 887.

[13] H. Chang, A. Krieger, A. Astolfi, E. Pistikopoulos, Robust multiparametric model predictive control for $\{L P V\}$ systems with application to anaesthesia, Journal of Process Control 24 (2014) 1538 - 1547.

[14] J. Lemos, D. Caiado, B. Costa, L. Paz, T. Mendonça, E. S. Rabiço, R., M. Seabra, Robust control of maintenance-phase anesthesia, IEEE Control Systems 14(6) (2014) 24-38.

[15] G. Dumont, Closed-loop control of anesthesia - a review, Proceedings of the 8th IFAC Symposium on Biological and Medical Systems (2012) (2012).

[16] F. N. Nogueira, T. Mendonça, P. Rocha, Controlling the depth of anesthesia by a novel positive control strategy, Computer Methods and Programs in Biomedicine. 114(3) (2014) e87-97.

[17] M. M. Silva, T. Mendonça, T. Wigren, Online nonlinear identification of the effect of drugs in anaesthesia using a minimal parameterization 
and bis measurements, Proceedings of the American Control Conference (ACC10) (2010) 4379-4384.

[18] C. F. Minto, T. W. Schnider, T. Short, K. Gregg, A. Gentilini, S. L. Shafer, Response surface model for anesthetic drug interactions., Anesthesiology 92 (2000) 1603-1616.

[19] T. Mendonça, H. Alonso, M. M. Silva, S. Esteves, M. Seabra, Comparing different identification approaches for the depth of anesthesia using bis measurements, 16th IFAC Symposium on System Identification (2012).

[20] J. P. LaSalle, The Stability of Dynamical Systems, SIAM, Bristol, England, 1976.

[21] F. N. Nogueira, T. Mendonça, P. Rocha, A new retuning approach for doa reference tracking improvement, Proceedings of The 19th World Congress of the International Federation of Automatic Control. (2014).

[22] B. Costa, L. Paz, D. Caiado, S. Esteves, R. Rabiço, M. Seabra, Z. Moreira, J. Lemos, T. Mendonça, Galeno: Computer aided system for modeling, monitoring and control in anesthesia, IEEE Journal of Biomedical and Health Informatics. (Submitted) (2014).

[23] C. M. Ionescu, R. D. Keyser, M. M. Struys, Evaluation of a propofol and remifentanil interaction model for predictive control of anesthesia induction, Proceedings of the 50th IEEE Conference on Decision and Control and European Control Conference (CDC-ECC) (2011).

[24] J. M. Bailey, W. M. Haddad, Drug dosing control in clinical pharmacology, Control Systems Magazine, IEEE 25 (2005) 35-51. 
[25] B. Marsh, N. Norton, G. N. Kenny, Pharmacokinetic model driven infusion of propofol in children, Br. J. Anaesthesia 67 (1991) 41-48.

[26] C. F. Minto, T. W. Schnider, T. D. Egan, E. Youngs, H. J. Lemmens, P. L. Gambus, V. Billard, J. F. Hoke, K. H. Moore, D. J. Hermann, K. T. Muir, J. W. Mandema, S. L. Shafer, Influence of age and gender on the pharmacokinetics and pharmacodynamics of remifentanil. i. model development, Anesthesiology 86 (1997) 10-23.

[27] T. W. Schnider, C. F. Minto, P. L. Gambus, C. Andresen, D. B. Goodale, S. L. Shafer, E. J. Youngs, The influence of method of administration and covariates on the pharmacokinetics of propofol in adult volunteers, Anesthesiology 8 (1998) 1170-1182. 